\title{
Triclosan resistance in clinical isolates of Acinetobacter baumannii
}

\author{
Correspondence \\ Yunsong $\mathrm{Yu}$ \\ yvys119@163.com
}

Received 4 December 2008

Accepted 5 May 2009

\author{
Yagang Chen, Borui Pi, Hua Zhou, Yunsong Yu and Lanjuan Li
}

State Key Laboratory for Diagnosis and Treatment of Infectious Disease, First Affiliated Hospital, College of Medicine, Zhejiang University, Hangzhou, Zhejiang 310003, PR China

\begin{abstract}
The susceptibility to triclosan of 732 clinical Acinetobacter baumannii isolates obtained from 25 hospitals in 16 cities in China from December 2004 to December 2005 was screened by using an agar dilution method. Triclosan MICs ranged between 0.015 and $16 \mathrm{mg} \mathrm{I}^{-1}$, and the $\mathrm{MIC}_{90}$ was $0.5 \mathrm{mg} \mathrm{l}^{-1}$, lower than the actual in-use concentration of triclosan. Twenty triclosan-resistant isolates (MICs $\geqslant 1 \mathrm{mg} \mathrm{l}^{-1}$ ) were characterized by antibiotic susceptibility, clonal relatedness, fabl mutation, fab/ expression, and efflux pump phenotype and expression to elucidate the resistance mechanism of $A$. baumannii to triclosan. The resistance rates of triclosan-resistant isolates to imipenem, levofloxacin, amikacin and tetracycline were higher than those of triclosan-sensitive isolates. Triclosan resistance was artificially classified as low level (MICs 1-2 $\mathrm{mg} \mathrm{I}^{-1}$ ) or high level $\left(\mathrm{MICs} \geqslant 4 \mathrm{mg} \mathrm{l}^{-1}\right)$. High-level triclosan resistance could be explained by a Gly95Ser mutation of Fabl, whilst wild-type fab/ was observed to be overexpressed in low-level resistant isolates. Active efflux did not appear to be a major reason for acquired triclosan resistance, but acquisition of resistance appeared to be dependent on a background of intrinsic triclosan efflux.
\end{abstract}

\section{INTRODUCTION}

Acinetobacter baumannii is a clinically significant pathogen that is characteristic of multidrug resistance, and has a great survival ability in environmental and human reservoirs (Peleg et al., 2008). Efflux is an important mechanism for the emergence of multidrug-resistant pathogens. Three multidrug efflux pumps have been identified in A. baumannii so far, namely AdeABC, AbeM and AdeIJK, which display broad substrate specificity including antibacterial agents, biocides and dyes (DamierPiolle et al., 2008; Magnet et al., 2001; Su et al., 2005).

Triclosan is a broad-spectrum biocide that has been used for over 30 years. In addition to health-care settings, it is widely used in hand soaps, toothpastes, shower gels, hand creams and underarm deodorants, as well as in fabrics and plastics (Yazdankhah et al., 2006). Like other biocides, triclosan has been thought to act as a non-specific biocide by affecting membrane structure and function. Recently, a defined target in the fatty acid biosynthetic pathway, enoyl-acyl carrier protein reductase (FabI), has been shown to be inhibited by triclosan in several studies (Heath et al., 1999; Hoang \& Schweizer, 1999; McMurry et al., 1998b). fabI mutations in active-site residues and its overexpression confer resistance to triclosan to varying degrees (Fan et al., 2002; Heath et al., 1999; McMurry et al., 1998b; Webber et al., 2008). In addition, triclosan is a substrate of multidrug efflux pumps, such as AcrAB-TolC in Escherichia coli, and MexAB-OprM,

Abbreviations: CCCP, carbonylcyanide-3-chlorophenyl hydrazone; $\mathrm{PA} \beta \mathrm{N}$, phenylarginine- $\beta$-naphthylamide.
MexCD-OprJ and MexEF-OprN in Pseudomonas aeruginosa. Active effluxes are responsible for intrinsic and acquired resistance to triclosan in many bacteria (Chuanchuen et al., 2001; McMurry et al., 1998a; Mima et al., 2007).

Triclosan resistance has been studied in several bacteria, such as E. coli, Staphylococcus aureus, P. aeruginosa and Salmonella enterica (Chuanchuen et al., 2001; Fan et al., 2002; Hoang \& Schweizer, 1999; McMurry et al., 1998a, b; Webber et al., 2008). However, characterization of triclosan resistance in A. baumannii has not yet been reported. In this study, we screened the susceptibility of 732 clinically derived A. baumannii isolates to triclosan. Further investigation of fabI mutation, fabI expression and efflux pump expression was performed to elucidate the resistance mechanism of A. baumannii to triclosan.

\section{METHODS}

Bacteria. A total of 732 A. baumannii isolates was obtained from 25 hospitals in 16 cities in China from December 2004 to December 2005. All isolates were identified as members of the Acinetobacter calcoaceticus-A. baumannii complex by using a Vitek $\mathrm{GNI}^{+}$card (bioMérieux). Species identification was confirmed by PCR amplification of the intrinsic bla $a_{\mathrm{OXA}-51}$ allele (Turton et al., 2006). A. baumannii ATCC $19606^{\mathrm{T}}$ was used as the reference strain.

Susceptibility test. MICs were determined by an agar dilution method according to Clinical and Laboratory Standards Institute guidelines. The drugs tested were triclosan (Sigma), imipenem (Merck), levofloxacin (Bayer), amikacin (Sigma) and tetracycline (Sigma). P. aeruginosa ATCC 27853 was used as a control. The MICs 
of triclosan and the four other antimicrobial agents were also determined in the presence of the efflux pump inhibitors phenylarginine- $\beta$-naphthylamide (PA $\beta \mathrm{N}$ ) (Sigma) at a concentration of $100 \mathrm{mg} \mathrm{l}^{-1}$ and carbonylcyanide-3-chlorophenyl hydrazone (CCCP) (Sigma) at a concentration of $100 \mu \mathrm{M}$.

PFGE. Genomic DNA was prepared from isolates and digested with ApaI (Sangon) as described by Seifert \& Gerner-Smidt (1995). DNA fragments were separated by electrophoresis in $1 \%$ agarose gels (Sangon) using a CHEF Mapper XA (Bio-Rad) at $14{ }^{\circ} \mathrm{C}$ and $6 \mathrm{~V} \mathrm{~cm}^{-1}$ with alternating pulses at a $120^{\circ}$ angle in a 5-20 s pulse time gradient for $22 \mathrm{~h}$. PFGE results were interpreted by using the criteria of Tenover et al. (1995).

PCR amplification and sequencing. The primers fabI_up and fabI_dw (Table 1) were used to amplify the complete fabI gene, in accordance with the genomic sequence of A. baumannii ATCC 17978 (GenBank accession no. NC_009085). The amplicons were sequenced using an ABI 3730 sequence analyser (Applied Biosystems). The nucleotide sequences and deduced amino acid sequences were analysed using MEGA software (version 4.0).

Real-time RT-PCR. Expression of the genes fabI, adeB, abeM and adeJ was measured by using real-time RT-PCR. The primers used are described in Table 1. Total RNA was prepared using an E.Z.N.A. bacterial RNA kit (Omega Bio-Tek) according to the manufacturer's instructions. The concentration and quality of RNA were determined using a spectrophotometer (Beckman Instruments). Reverse transcription was performed by using a PrimeScript RT reagent kit (Takara Bio) with 300 ng RNA in a $10 \mu \mathrm{l}$ reaction mixture containing $2 \mu \mathrm{l}$ PrimeScript buffer, $0.5 \mu \mathrm{l}$ PrimeScript reverse transcriptase enzyme mix I, $0.5 \mu \mathrm{l}$ oligo $(\mathrm{dT})$ primer $(50 \mu \mathrm{M})$ and $0.5 \mu \mathrm{l}$ random hexamers $(100 \mu \mathrm{M})$. The mixtures were incubated at $37^{\circ} \mathrm{C}$ for $40 \mathrm{~min}$ to synthesize cDNA and heated to $85{ }^{\circ} \mathrm{C}$ for $10 \mathrm{~s}$ to inactivate the reverse transcriptase. A DNA engine Opticon 2 real-time PCR detection system (Bio-Rad) was used for real-time PCR. PCRs were performed by using a SYBR PrimeScript RTPCR kit (Takara Bio) with $1 \mu \mathrm{l}$ cDNA and $0.5 \mu$ each primer $(10 \mu \mathrm{M})$ in a total volume of $25 \mu$ l. Each sample was run in duplicate. The $16 \mathrm{~S}$ rRNA gene was used as a housekeeping gene to normalize the expression of target genes. Results were given as the relative expression of the mRNA compared with that of A. baumannii ATCC $19606^{\mathrm{T}}$, which was set at 1 .

\section{RESULTS}

Triclosan MICs against 732 clinical A. baumannii isolates ranged from 0.015 to $16 \mathrm{mg} \mathrm{l}^{-1}$ with a $\mathrm{MIC}_{50}$ of $0.25 \mathrm{mg}$ $\mathrm{l}^{-1}$ and a $\mathrm{MIC}_{90}$ of $0.5 \mathrm{mg} \mathrm{l}^{-1}$, which are lower than the actual in-use concentrations of triclosan (2000-20000 mg $\left.1^{-1}\right)$. However, triclosan is stable in the environment and may remain on surfaces at low concentrations. Materials that release triclosan slowly, such as plastics and fabrics, are also used at low concentrations. For example, the final concentration of triclosan in triclosan baths is approximately 4-5 $\mathrm{mg} \mathrm{l}^{-1}$, lower than the MICs of some clinical isolates (Bamber \& Neal, 1999). In this study, we found 20 isolates with elevated triclosan MICs (MICs $>\mathrm{MIC}_{90}$; MICs $\geqslant 1 \mathrm{mg} \mathrm{l}^{-1}$ ), and the least susceptible isolates had MICs of up to $16 \mathrm{mg} \mathrm{l}^{-1}$. The clinical implication of this reduced susceptibility to triclosan is unknown.

The 20 isolates with reduced susceptibility to triclosan were artificially classified into two groups, low-level resistance (MICs $1-2 \mathrm{mg} \mathrm{l}^{-1}, n=8$ ) and high-level resistance (MICs $\geqslant 4 \mathrm{mg} \mathrm{l}^{-1}, n=12$ ) (Table 2). Twenty randomly selected sensitive isolates and ATCC $19606^{\mathrm{T}}$ were used as triclosansensitive control isolates to test for common features found only among the resistant isolates. The resistant isolates showed two distinct PFGE patterns, of which three isolates belonged to pulsotype $\mathrm{A}$ and 17 isolates belonged to pulsotype B (data not shown). The susceptibility to four different antimicrobial agents (amikacin, tetracycline, levofloxacin and imipenem) was compared between the triclosan-resistant and -sensitive isolates (Table 2). The MICs of the four antimicrobial agents were interpreted using Clinical and Laboratory Standards Institute criteria. All triclosan-resistant isolates were also resistant to all four antimicrobial agents, whilst the rates of resistance to amikacin, tetracycline, levofloxacin and imipenem amongst the triclosan-sensitive isolates were 55, 55, 40 and $40 \%$, respectively. There were statistically significant differences in antimicrobial susceptibility between the two groups of isolates by $\chi^{2}$ test $(P<0.05$, two-tailed test). Imipenem MICs were reduced by $\mathrm{PA} \beta \mathrm{N}$ in imipenem-resistant isolates; however, no or few inhibitory effects were observed on the MICs of amikacin, tetracycline and levofloxacin in the presence of $\mathrm{PA} \beta \mathrm{N}$ or CCCP. Previous

Table 1. Sequences of primers used for this study

\begin{tabular}{|c|c|c|c|}
\hline Gene & Primer & Sequence $\left(5^{\prime} \rightarrow 3^{\prime}\right)$ & Reference \\
\hline$f a b I$ & fabI_dw & CTGAAGTCCGCTACCGTTAT & \\
\hline fabI (quantitative) & fabI_Q_dw & GCACACGCTTGATGGTGACT & \\
\hline \multirow[t]{2}{*}{$a d e B$ (quantitative) } & adeB_Q_up & AACGGACGACCATCTTTGAGTATT & Peleg et al. (2007) \\
\hline & adeB_Q_dw & CAGTTGTTCCATTTCACGCATT & \\
\hline \multirow{2}{*}{ adeJ (quantitative) } & adeJ_Q_up & AGCGTATTGACAGCGGTATT & This study \\
\hline & adeJ_Q_dw & ATAGAGCACGCCAGAGAAGA & \\
\hline \multirow[t]{2}{*}{ 16S rRNA (quantitative) } & 16S RNA_Q_up & CAGCTCGTGTCGTGAGATGT & Peleg et al. (2007) \\
\hline & 16S RNA_Q_dw & CGTAAGGGCCATGATGACTT & \\
\hline
\end{tabular}


Numbers in parentheses refer to fold changes in triclosan susceptibility.

\begin{tabular}{|c|c|c|c|c|c|c|c|c|c|c|c|c|c|c|c|c|c|c|c|c|}
\hline \multirow[t]{2}{*}{ Strain } & \multicolumn{15}{|c|}{$\operatorname{MIC}\left(\mathrm{mg} \mathrm{l}^{-1}\right)$} & \multicolumn{2}{|c|}{ FabI } & \multicolumn{3}{|c|}{$\begin{array}{c}\text { Relative efflux } \\
\text { pump expression }\end{array}$} \\
\hline & TRI & $\begin{array}{l}\text { TRI+ } \\
\text { PA/N }\end{array}$ & $\begin{array}{l}\text { TRI }+ \\
\text { CCCP }\end{array}$ & IMP & $\begin{array}{l}\text { IMP+ } \\
\text { PA/ } \beta \text { N }\end{array}$ & $\begin{array}{l}\text { IMP + } \\
\text { CCCP }\end{array}$ & LEV & $\begin{array}{l}\text { LEV + } \\
\text { PA/N }\end{array}$ & $\begin{array}{l}\text { LEV + } \\
\text { CCCP }\end{array}$ & AMK & $\begin{array}{c}\text { AMK + } \\
\text { PA/N }\end{array}$ & $\begin{array}{c}\mathrm{AMK}+ \\
\mathrm{CCCP}\end{array}$ & TET & $\begin{array}{l}\text { TET + } \\
\text { PA/N }\end{array}$ & $\begin{array}{l}\text { TET + } \\
\text { CCCP }\end{array}$ & Mutation & $\begin{array}{c}\text { Relative } \\
\text { expression }\end{array}$ & $a d e B$ & abeM & adeJ \\
\hline H6 & 16 & $4(4)$ & $8(2)$ & $>256$ & $>128(-)$ & $>128(-)$ & 32 & $8(4)$ & $8(4)$ & 256 & $128(2)$ & $128(2)$ & 1024 & $512(2)$ & $1024(1)$ & Gly95Ser & 0.60 & 0.01 & 0.22 & 1.40 \\
\hline F6 & 4 & $2(2)$ & $4(1)$ & 64 & $16(4)$ & $32(2)$ & 16 & $16(1)$ & $16(1)$ & 512 & $512(1)$ & $512(1)$ & 1024 & $1024(1)$ & $1024(1)$ & Gly95Ser & 4.35 & 0.72 & 4.15 & 3.13 \\
\hline W398 & 4 & $2(2)$ & $4(1)$ & 64 & $16(4)$ & $32(2)$ & 16 & $16(1)$ & $16(1)$ & 1024 & $1024(1)$ & $1024(1)$ & 1024 & $512(2)$ & $512(2)$ & Gly95Ser & 1.46 & 0.92 & 1.42 & 2.00 \\
\hline F1 & 4 & $1(4)$ & $2(2)$ & 64 & $16(4)$ & $32(2)$ & 32 & $16(2)$ & $16(2)$ & 512 & $512(1)$ & $512(1)$ & 1024 & 1024 & $1024(1)$ & Gly95Ser & 3.34 & 0.21 & 4.68 & 1.41 \\
\hline F2 & 4 & $1(4)$ & $4(1)$ & 64 & $16(4)$ & $32(2)$ & 32 & $16(2)$ & $16(2)$ & 512 & $512(1)$ & $512(1)$ & 1024 & $512(2)$ & $1024(1)$ & Gly95Ser & 4.48 & 0.62 & 0.77 & 1.07 \\
\hline F30 & 4 & $1(4)$ & $2(2)$ & 32 & $8(4)$ & $16(2)$ & 8 & $8(1)$ & $8(1)$ & 256 & $128(2)$ & $128(2)$ & 512 & $128(4)$ & $256(2)$ & Gly95Ser & 1.45 & 0.00 & 0.96 & 1.32 \\
\hline $\mathrm{H} 1$ & 4 & $1(4)$ & $4(1)$ & 64 & $16(4)$ & $32(2)$ & 8 & $8(1)$ & $8(1)$ & 128 & $64(2)$ & $128(1)$ & 1024 & $1024(1)$ & $256(4)$ & Gly95Ser & 2.06 & 0.00 & 4.91 & 1.44 \\
\hline $\mathrm{H} 7$ & 4 & $1(4)$ & $4(1)$ & 128 & $16(8)$ & $32(4)$ & 32 & $16(2)$ & $16(2)$ & 1024 & $1024(1)$ & $1024(1)$ & 1024 & 1024 (1) & 1024 & Gly95Ser & 1.40 & 0.99 & 2.06 & 2.37 \\
\hline W391 & 4 & $1(4)$ & $4(1)$ & 128 & $32(4)$ & $64(2)$ & 16 & $16(1)$ & $16(1)$ & 1024 & $1024(1)$ & $1024(1)$ & 1024 & 1024 (1) & $1024(1)$ & Gly95Ser & 0.94 & 0.50 & 0.77 & 1.78 \\
\hline W396 & 4 & $1(4)$ & $4(1)$ & 64 & $16(4)$ & $32(2)$ & 16 & $16(1)$ & $16(1)$ & 1024 & $1024(1)$ & $1024(1)$ & 1024 & $512(2)$ & $1024(1)$ & Gly95Ser & 0.63 & 1.12 & 2.37 & 2.28 \\
\hline W378 & 4 & $2(2)$ & $2(1)$ & 128 & $32(4)$ & $64(2)$ & 16 & $16(1)$ & $16(1)$ & 1024 & $1024(1)$ & $1024(1)$ & 1024 & 1024 & $1024(1)$ & Gly95Ser & 0.86 & 0.23 & 0.89 & 1.73 \\
\hline NB207 & 4 & $1(4)$ & $2(1)$ & 128 & $64(2)$ & $64(2)$ & 8 & $8(1)$ & $8(1)$ & 512 & $512(1)$ & $512(1)$ & 1024 & $512(2)$ & $1024(1)$ & Gly95Ser & 2.24 & 0.88 & 6.30 & 2.42 \\
\hline F27 & 2 & $0.25(8)$ & $1(2)$ & 64 & $8(8)$ & $8(8)$ & 32 & $16(2)$ & $16(2)$ & 1024 & $1024(1)$ & $1024(1)$ & 1024 & 1024 & $1024(1)$ & - & 6.88 & 0.29 & 3.93 & 1.04 \\
\hline F29 & 2 & $0.25(8)$ & $1(2)$ & 64 & $8(8)$ & $16(4)$ & 32 & $16(2)$ & $16(2)$ & 1024 & $1024(1)$ & $1024(1)$ & 1024 & $1024(1)$ & $512(2)$ & - & 8.00 & 0.73 & 2.44 & 2.91 \\
\hline F13 & 1 & $0.125(8)$ & $1(1)$ & 128 & $32(4)$ & $32(4)$ & 32 & $16(2)$ & $16(2)$ & 1024 & $1024(1)$ & $1024(1)$ & 1024 & 1024 & $1024(1)$ & - & 7.09 & 0.62 & 2.82 & 2.10 \\
\hline F16 & 1 & $0.125(8)$ & $1(1)$ & 64 & $32(2)$ & $32(2)$ & 32 & $16(2)$ & $16(2)$ & 1024 & $1024(1)$ & $1024(1)$ & 1024 & 1024 & $1024(1)$ & - & 8.46 & 0.61 & 11.25 & 2.64 \\
\hline F17 & 1 & $0.125(8)$ & $1(1)$ & 64 & $32(2)$ & $32(2)$ & 32 & $16(2)$ & $16(2)$ & 1024 & $1024(1)$ & $1024(1)$ & 1024 & $512(2)$ & $1024(1)$ & - & 7.77 & 1.08 & 2.02 & 2.28 \\
\hline F22 & 1 & $0.125(8)$ & $1(1)$ & 128 & $32(4)$ & $64(2)$ & 16 & $16(1)$ & $16(1)$ & 1024 & $1024(1)$ & $1024(1)$ & 1024 & $1024(1)$ & 1024 & - & 9.72 & 1.24 & 1.46 & 2.11 \\
\hline F12 & 1 & $0.06(16)$ & $1(1)$ & 256 & $64(4)$ & $128(2)$ & 32 & $16(2)$ & $16(2)$ & 1024 & $1024(1)$ & $1024(1)$ & 1024 & 1024 & $1024(1)$ & - & 11.46 & 0.31 & 6.19 & 1.89 \\
\hline F23 & 1 & $0.06(16)$ & $1(1)$ & 128 & $32(4)$ & $64(2)$ & 32 & $16(2)$ & $16(2)$ & 1024 & $1024(1)$ & $1024(1)$ & 1024 & $1024(1)$ & $1024(1)$ & - & 9.35 & 1.34 & 1.56 & 2.08 \\
\hline $\mathrm{ZY} 2$ & 0.5 & $0.25(2)$ & $0.5(1)$ & 128 & $32(4)$ & $64(2)$ & 16 & $8(2)$ & $8(2)$ & 512 & $512(1)$ & $256(2)$ & 512 & $256(2)$ & $512(1)$ & - & 0.80 & 0.28 & 0.69 & 0.31 \\
\hline F15 & 0.5 & $0.06(8)$ & 0.125 & 128 & $16(8)$ & $32(4)$ & 16 & $4(4)$ & $4(4)$ & 1024 & $1024(1)$ & $1024(1)$ & 512 & $512(1)$ & $512(1)$ & - & 0.96 & 0.35 & 2.69 & 0.40 \\
\hline $\mathrm{ZY} 43$ & 0.25 & $0.03(8)$ & $0.25(1)$ & 128 & $32(4)$ & $64(2)$ & 8 & $8(1)$ & $8(1)$ & 1024 & $1024(1)$ & $1024(1)$ & 512 & $256(2)$ & $512(1)$ & - & 0.71 & 0.43 & 3.65 & 0.97 \\
\hline ZY42 & 0.25 & $0.015(16)$ & 0.125 & 128 & $32(4)$ & $64(2)$ & 8 & $4(2)$ & $4(2)$ & 512 & $256(1)$ & $512(1)$ & 512 & $512(1)$ & $512(1)$ & - & 0.95 & 0.00 & 1.57 & 0.28 \\
\hline ZY47 & 0.25 & $0.004(64)$ & 0.125 & 0.25 & $0.25(1)$ & $0.25(1)$ & 0.06 & $0.06(1)$ & $0.06(1)$ & 256 & $256(1)$ & $256(1)$ & 2 & $2(1)$ & $2(1)$ & - & 0.97 & 0.24 & 0.74 & 0.68 \\
\hline CQ5 & 0.25 & $0.03(8)$ & $0.125(2)$ & 4 & $1(4)$ & $2(2)$ & 8 & $4(2)$ & $4(2)$ & 512 & $512(1)$ & $512(1)$ & 512 & $512(1)$ & $512(1)$ & - & 1.47 & 1.00 & 3.12 & 0.40 \\
\hline CQ6 & 0.25 & $0.03(8)$ & $0.25(1)$ & 4 & $4(1)$ & $4(1)$ & 8 & $4(2)$ & $4(2)$ & 1024 & $1024(1)$ & $1024(1)$ & 1024 & $512(2)$ & $512(2)$ & - & 0.95 & 3.41 & 1.59 & 0.83 \\
\hline SY206 & 0.25 & $0.03(8)$ & $0.25(1)$ & 128 & $16(8)$ & $64(2)$ & 8 & $8(1)$ & $8(1)$ & 1024 & $1024(1)$ & $1024(1)$ & 1024 & $512(2)$ & $512(2)$ & - & 2.48 & 1.00 & 3.10 & 0.84 \\
\hline W1657 & 0.125 & $0.03(4)$ & $0.06(2)$ & 0.5 & $0.25(2)$ & $0.25(2)$ & 0.25 & $0.25(1)$ & $0.125(2)$ & 2 & $2(1)$ & $2(1)$ & 2 & $1(2)$ & $2(1)$ & - & 1.26 & 0.04 & 0.39 & 0.84 \\
\hline L9924 & 0.125 & $0.03(4)$ & $0.06(2)$ & 0.5 & $0.25(2)$ & $0.25(2)$ & 0.125 & 0.125 & $0.06(2)$ & 2 & $2(1)$ & $2(1)$ & 2 & $2(1)$ & $1(2)$ & - & 0.24 & 0.18 & 3.31 & 0.81 \\
\hline JX16 & 0.125 & $0.03(4)$ & $0.125(1)$ & 0.5 & $0.25(2)$ & $0.25(2)$ & 0.5 & $0.25(2)$ & $0.25(2)$ & 2 & $2(1)$ & $2(1)$ & 2 & $2(1)$ & $2(1)$ & - & 0.47 & 0.42 & 0.01 & 0.43 \\
\hline B30 & 0.125 & $0.03(4)$ & $0.125(1)$ & 0.5 & $0.25(2)$ & $0.25(2)$ & 0.25 & $0.125(2)$ & $0.125(2)$ & 4 & $4(1)$ & $4(1)$ & 2 & $2(1)$ & $1(2)$ & - & 1.47 & 0.26 & 4.40 & 4.15 \\
\hline SH46 & 0.125 & $0.03(4)$ & $0.125(1)$ & 0.25 & $0.25(1)$ & $0.25(1)$ & 0.125 & 0.125 & $0.125(1)$ & 1 & $1(1)$ & $1(1)$ & 2 & $2(1)$ & $1(2)$ & - & 1.32 & 2.38 & 1.57 & 0.35 \\
\hline F11 & 0.125 & $0.008(16)$ & 0.125 & 64 & $16(4)$ & $32(2)$ & 4 & $4(1)$ & $4(1)$ & 1024 & $1024(1)$ & $1024(1)$ & 512 & $512(1)$ & $512(1)$ & - & 1.48 & 1.31 & 4.99 & 0.51 \\
\hline H18 & 0.125 & 0.015 & 0.125 & 128 & $32(4)$ & $64(2)$ & 8 & $4(2)$ & $4(2)$ & 1024 & $1024(1)$ & $1024(1)$ & 512 & $512(1)$ & $512(1)$ & - & 1.15 & 0.44 & 4.92 & 2.66 \\
\hline
\end{tabular}




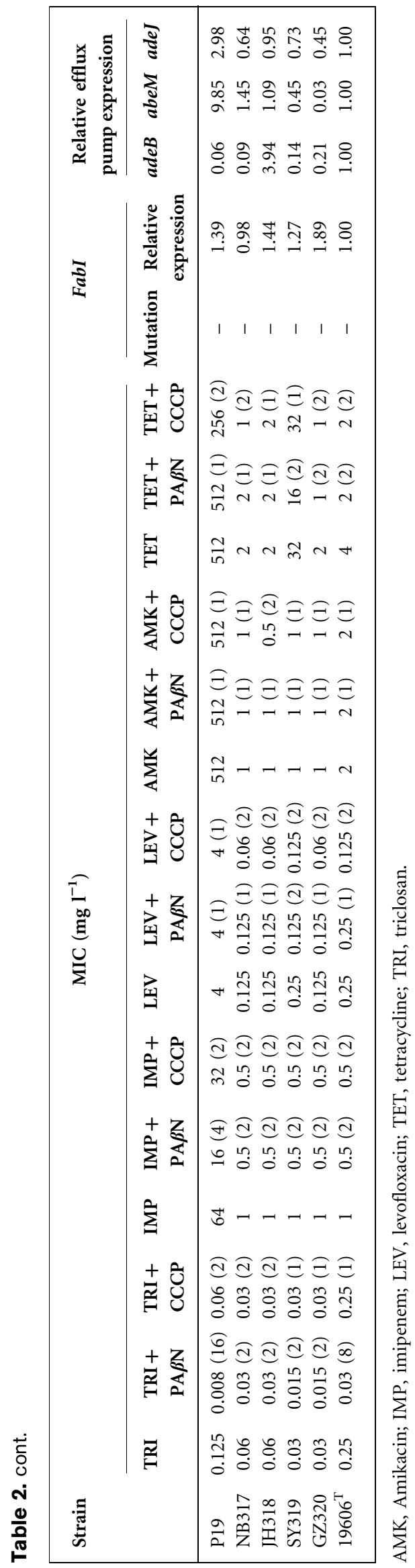

studies have also demonstrated that the inhibitive effects of $\mathrm{PA} \beta \mathrm{N}$ are limited to nalidixic acid, clarithromycin, rifampicin and linezolid in A. baumannii (Pannek et al., 2006; Ribera et al., 2002).

Twelve high-level resistant isolates had substitutions of glycine (GGC) to serine (AGC) at aa 95 in FabI. This missense mutation was found in all of the high-level resistant isolates but not in any of the low-level resistant ones (Table 2). Gly95 is located at a signature motif for the FAD-dependent pyridine nucleotide reductase family [D..(FILMV)..(IV)G..P...L], which is equivalent to the glycine found at aa 93 of $E$. coli and that at aa 95 of $P$. aeruginosa (Heath et al., 1999; Hoang \& Schweizer, 1999). Gly93Ser and Gly93Val substitutions in E. coli and a Gly95Val substitution in $P$. aeruginosa significantly elevate triclosan MICs and confer resistance to triclosan by preventing the formation of the FabI-NAD ${ }^{+}$-triclosan ternary complex (Bergler et al., 1992; Heath et al., 1999; Hoang \& Schweizer, 1999). Heath et al. (1999) found that Gly93 in E. coli lies on one side of the substrate-binding pocket; when Val replaces Gly93, it occupies the space normally occupied by the dichlorophenyl ring of triclosan; they suggested that the Gly93Val mutation thus hampered formation of the ternary complex due to steric interference. We hypothesized that the Gly95Ser substitution in FabI of $A$. baumannii might result in triclosan resistance by the same mechanism. Because the mutant fabI gene was expressed normally or was slightly increased, high levels of triclosan resistance can be attributed mainly to fabI mutation rather than fabI overexpression. However, for the low-level resistant isolates, expression of wild-type fabI was increased significantly and was higher than that in the high-level resistant isolates and the sensitive isolates, indicating that low-level resistance was achieved via overexpression of wildtype fabI (Fig. 1). FabI overexpression has also been observed in an intermediate resistant clinical isolate of Staphylococcus aureus (Fan et al., 2002). Moreover, low-level triclosan resistance has been observed when wild-type fabI was overexpressed artificially in Salmonella enterica serovar Typhimurium (Webber et al., 2008).

Efflux pump overexpression has been shown to account for triclosan resistance in micro-organisms such as $P$. aeruginosa and E. coli (Chuanchuen et al., 2001; McMurry et al., 1998a; Mima et al., 2007; Webber et al., 2008). Su et al. (2005) confirmed that triclosan was a substrate for the efflux pump AbeM in A. baumannii, which increased the triclosan MIC fourfold (from 0.5 to $2 \mathrm{mg}^{-1}$ ).

In this study, efflux pump inhibition tests and expression studies were performed to demonstrate the relationship between active efflux and triclosan resistance. Triclosan MICs were determined in the presence or absence of two types of efflux pump inhibitor, PA $\beta \mathrm{N}$ and CCCP (Table 2). CCCP exhibited few or no inhibitory effects on triclosan MICs at a concentration of $100 \mu \mathrm{M}$. In contrast, PA $\beta \mathrm{N}$ inhibited triclosan MICs in both triclosan-sensitive and -resistant isolates. Triclosan MICs were reduced more than 


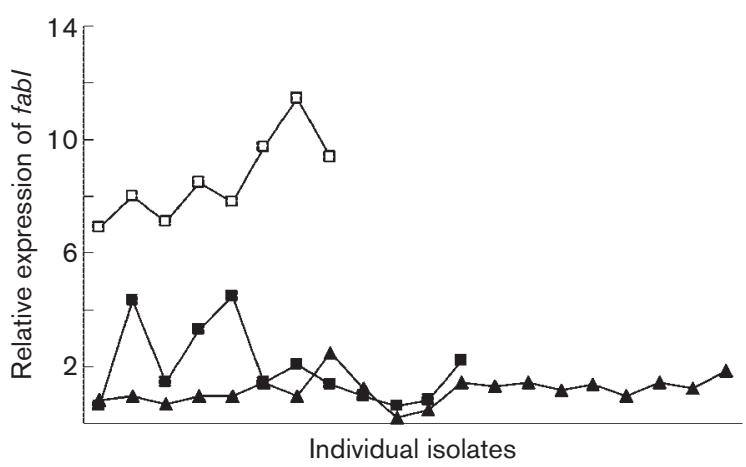

Fig. 1. Comparison of the relative fabl expression of triclosanresistant and -sensitive isolates. From left to right, the 12 high-level resistant isolates, 8 low-level resistant isolates and 20 sensitive isolates represented are (respectively): $\mathrm{H6}$, F6, W398, F1, F2, F30, $\mathrm{H} 1, \mathrm{H} 7$, W391, W396, W378 and NB207; F27, F29, F13, F16, F17, F22, F12 and F23; and ZY2, F15, ZY43, ZY42, ZY47, CQ5, CQ6, SY206, W1657, L9924, JX16, B30, SH46, F11, H18, P19, NB317, JH318, SY319 and GZ320. - , High-level resistant isolates; $\square$, low-level resistant isolates; $\boldsymbol{\Lambda}$, sensitive isolates.

fourfold in the presence of $\mathrm{PA} \beta \mathrm{N}$ in 15 triclosan-sensitive isolates and 17 triclosan-resistant isolates, which was taken as a sign of efflux pump overexpression. The most significant reduction in triclosan MICs was 64-fold. The susceptibility to triclosan of 12 isolates with a Gly95Ser mutation in the presence of $\mathrm{PA} \beta \mathrm{N}$ was not restored to the level of sensitive isolates - they were still resistant to triclosan $\left(\mathrm{MICs} \geqslant 1 \mathrm{mg} \mathrm{l}^{-1}\right)$. PA $\beta \mathrm{N}$ affects the MICs of antimicrobial agents by two different mechanisms, inhibiting efflux pumps and enhancing membrane permeability (Pannek et al., 2006). If $\mathrm{PA} \beta \mathrm{N}$ reduced the triclosan MICs by inhibiting the efflux pumps, active efflux may be responsible for the intrinsic MIC of triclosan against A. baumannii, and lowlevel and high-level resistance to triclosan might be acquired by $f a b I$ overexpression and fabI mutation, respectively, against a background of intrinsic triclosan efflux.

The genes adeB, abeM and adeJ encode the resistancenodulation-cell division (RND) protein of the efflux pumps AdeABC, AbeM and AdeIJK, respectively. As ade $A B C$ genes are co-transcribed, the real-time RT-PCR results for $a d e B$ represented expression of the AdeABC pump (Marchand et al., 2004), as for AdeIJK pumps (Damier-Piolle et al., 2008). Detailed information on the relative expression of adeB, abeM and adeJ genes is given in Table 2. No overexpression of the $a d e B$ gene was found in triclosan-resistant isolates; however, the abeM gene was found to be overexpressed in both triclosan-sensitive and -resistant isolates relative to the type strain ATCC $19606^{\mathrm{T}}$. No statistically significant differences in mean expression of $a d e B$ and $a b e M$ were found between triclosan-sensitive and -resistant isolates by one-way ANOVA $(P=0.491$ and 0.471 , respectively). Thus, it appeared that expression of the $a d e B$ and $a b e M$ genes did not correlate with triclosan

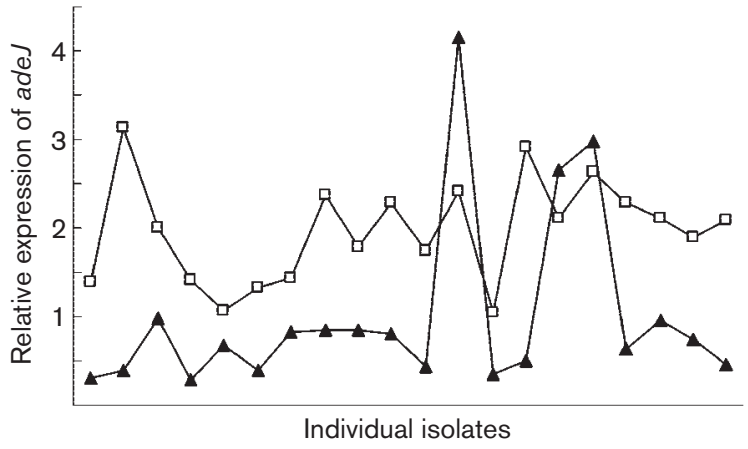

Fig. 2. Comparison of the relative adeJ expression of triclosanresistant and -sensitive isolates. From left to right, the 20 resistant isolates and 20 sensitive isolates represented are (respectively): H6, F6, W398, F1, F2, F30, H1, H7, W391, W396, W378, 207, F27, F29, F13, F16, F17, F22, F12 and F23; and ZY2, F15, ZY43, ZY42, ZY47, CQ5, CQ6, SY206, W1657, L9924, JX16, B30, SH46, F11, H18, P19, NB317, JH318, SY319 and GZ320. $\square$, Triclosan-resistant isolates; $\boldsymbol{\Delta}$, triclosan-sensitive isolates.

resistance. Comparison of adeJ expression between triclosan-sensitive and -resistant isolates is shown in Fig. 2. Although the mean expression level of adeJ in triclosanresistant isolates was higher than that in triclosan-sensitive isolates by one-way ANOVA $(P=0.001)$, the highest level of expression of the adeJ gene was found in the triclosansensitive isolate B30. No tight correlation could be found between expression of the three known efflux pumps (AdeABC, AbeM and AdeIJK) and triclosan resistance. However, we could not exclude the possibility that active efflux is one of the mechanisms for triclosan resistance, as a number of other putative drug transporters have been identified by whole-genome sequencing of $A$. baumannii strains AYE and SDF and their function remains to be verified (Fournier et al., 2006).

In summary, the majority of our clinical A. baumannii isolates from China were susceptible to triclosan, and only $3 \%$ exhibited reduced susceptibility. The resistance rates of the triclosan-resistant isolates to imipenem, levofloxacin, amikacin and tetracycline were higher than those of triclosan-sensitive isolates. High-level triclosan resistance could be explained by a Gly95Ser mutation of FabI, whilst overexpression of wild-type $f a b I$ was responsible for the low-level resistance. Active efflux did not appear to be a major reason for acquired triclosan resistance. As clinical isolates could acquire resistance to triclosan by fabI mutation and fabI overexpression, it is necessary to rationalize triclosan usage and monitor the susceptibility of $A$. baumannii to this drug.

\section{ACKNOWLEDGEMENTS}

This work was supported in part by a grant from the High Technology Research and Development Program of China (863 
program, no. 2006AA02Z413) and the Natural Science Foundation of Zhejiang Province (no. Y206123).

\section{REFERENCES}

Bamber, A. I. \& Neal, T. J. (1999). An assessment of triclosan susceptibility in methicillin-resistant and methicillin-sensitive Staphylococcus aureus. J Hosp Infect 41, 107-109.

Bergler, H., Hogenauer, G. \& Turnowsky, F. (1992). Sequences of the envM gene and of two mutated alleles in Escherichia coli. J Gen Microbiol 138, 2093-2100.

Chuanchuen, R., Beinlich, K., Hoang, T. T., Becher, A., KarkhoffSchweizer, R. R. \& Schweizer, H. P. (2001). Cross-resistance between triclosan and antibiotics in Pseudomonas aeruginosa is mediated by multidrug efflux pumps: exposure of a susceptible mutant strain to triclosan selects $n f x B$ mutants overexpressing MexCD-OprJ. Antimicrob Agents Chemother 45, 428-432.

Damier-Piolle, L., Magnet, S., Brémont, S., Lambert, T. \& Courvalin, P. (2008). AdeIJK, a resistance-nodulation-cell division pump effluxing multiple antibiotics in Acinetobacter baumannii. Antimicrob Agents Chemother 52, 557-562.

Fan, F., Yan, K., Wallis, N. G., Reed, S., Moore, T. D., Rittenhouse, S. F., DeWolf, W. E., Jr, Huang, J., McDevitt, D. \& other authors (2002). Defining and combating the mechanisms of triclosan resistance in clinical isolates of Staphylococcus aureus. Antimicrob Agents Chemother 46, 3343-3347.

Fournier, P. E., Vallenet, D., Barbe, V., Audic, S., Ogata, H., Poirel, L., Richet, H., Robert, C., Mangenot, S. \& other authors (2006). Comparative genomics of multidrug resistance in Acinetobacter baumannii. PLoS Genet 2, e7.

Heath, R. J., Rubin, J. R., Holland, D. R., Zhang, E., Snow, M. E. \& Rock, C. O. (1999). Mechanism of triclosan inhibition of bacterial fatty acid synthesis. J Biol Chem 274, 11110-11114.

Hoang, T. T. \& Schweizer, H. P. (1999). Characterization of Pseudomonas aeruginosa enoyl-acyl carrier protein reductase (FabI): a target for the antimicrobial triclosan and its role in acylated homoserine lactone synthesis. J Bacteriol 181, 5489-5497.

Magnet, S., Courvalin, P. \& Lambert, T. (2001). Resistancenodulation-cell division-type efflux pump involved in aminoglycoside resistance in Acinetobacter baumannii strain BM4454. Antimicrob Agents Chemother 45, 3375-3380.

Marchand, I., Damier-Piolle, L., Courvalin, P. \& Lambert, T. (2004). Expression of the RND-type efflux pump AdeABC in Acinetobacter baumannii is regulated by the AdeRS two-component system. Antimicrob Agents Chemother 48, 3298-3304.
McMurry, L. M., Oethinger, M. \& Levy, S. B. (1998a). Overexpression of marA, soxS, or acr $A B$ produces resistance to triclosan in laboratory and clinical strains of Escherichia coli. FEMS Microbiol Lett 166, 305-309.

McMurry, L. M., Oethinger, M. \& Levy, S. B. (1998b). Triclosan targets lipid synthesis. Nature 394, 531-532.

Mima, T., Joshi, S., Gomez-Escalada, M. \& Schweizer, H. P. (2007). Identification and characterization of TriABC-OpmH, a triclosan efflux pump of Pseudomonas aeruginosa requiring two membrane fusion proteins. J Bacteriol 189, 7600-7609.

Pannek, S., Higgins, P. G., Steinke, P., Jonas, D., Akova, M., Bohnert, J. A., Seifert, H. \& Kern, W. V. (2006). Multidrug efflux inhibition in Acinetobacter baumannii: comparison between 1-(1-naphthylmethyl)piperazine and phenyl-arginine- $\beta$-naphthylamide. $J$ Antimicrob Chemother 57, 970-974.

Peleg, A. Y., Adams, J. \& Paterson, D. L. (2007). Tigecycline efflux as a mechanism for nonsusceptibility in Acinetobacter baumannii. Antimicrob Agents Chemother 51, 2065-2069.

Peleg, A. Y., Seifert, H. \& Paterson, D. L. (2008). Acinetobacter baumannii: emergence of a successful pathogen. Clin Microbiol Rev 21, 538-582.

Ribera, A., Ruiz, J., Jiminez de Anta, M. T. \& Vila, J. (2002). Effect of an efflux pump inhibitor on the MIC of nalidixic acid for Acinetobacter baumannii and Stenotrophomonas maltophilia clinical isolates. J Antimicrob Chemother 49, 697-698.

Seifert, H. \& Gerner-Smidt, P. (1995). Comparison of ribotyping and pulsed-field gel electrophoresis for molecular typing of Acinetobacter isolates. J Clin Microbiol 33, 1402-1407.

Su, X. Z., Chen, J., Mizushima, T., Kuroda, T. \& Tsuchiya, T. (2005). AbeM, an $\mathrm{H}^{+}$-coupled Acinetobacter baumannii multidrug efflux pump belonging to the MATE family of transporters. Antimicrob Agents Chemother 49, 4362-4364.

Tenover, F. C., Arbeit, R. D., Goering, R. V., Mickelsen, P. A., Murray, B. E., Persing, D. H. \& Swaminathan, B. (1995). Interpreting chromosomal DNA restriction patterns produced by pulsed-field gel electrophoresis: criteria for bacterial strain typing. J Clin Microbiol 33, 2233-2239.

Turton, J. F., Woodford, N., Glover, J., Yarde, S., Kaufmann, M. E. \& Pitt, T. L. (2006). Identification of Acinetobacter baumannii by detection of the $b l a_{\text {OXA-51-like }}$ carbapenemase gene intrinsic to this species. J Clin Microbiol 44, 2974-2976.

Webber, M. A., Randall, L. P., Cooles, S., Woodward, M. J. \& Piddock, L. J. (2008). Triclosan resistance in Salmonella enterica serovar Typhimurium. J Antimicrob Chemother 62, 83-91.

Yazdankhah, S. P., Scheie, A. A., Hoiby, E. A., Lunestad, B. T., Heir, E., Fotland, T. O., Naterstad, K. \& Kruse, H. (2006). Triclosan and antimicrobial resistance in bacteria: an overview. Microb Drug Resist 12, 83-90. 